\title{
Influence of wikipedia and other web resources on acute and critical care decisions. a web-based surveY
}

\author{
B Rössler ${ }^{1 *}, \mathrm{H}$ Holldack ${ }^{2}$, K Schebesta $^{1}$ \\ From ESICM LIVES 2015 \\ Berlin, Germany. 3-7 October 2015
}

\section{Introduction}

Physicians use the Internet to gather medical information. However, little is known about the use and influence of Wikipedia, Google and other non-scientific web resources in acute and critical care medicine.

\section{Objectives}

Therefore, we conducted an online survey among anaesthetists and critical care providers to address the use of non-scientific web resources and their influence on decisions made.

\section{Methods}

After approval by the research ethics boards of the collaborating centres, 1,124 members of the ÖGARI (Austrian Society of Anaesthesiology, Resuscitation and Intensive Care) and 953 members of ANZCA (Australian and New Zealand College of Anaesthetists) were invited to participate in this anonymous online survey.

Demographic data as well as previous use of web-based resources were collected. The overall impact of online media on decisions made in an acute and critical care setting were assessed using 5 point Likert-like scales and multiple-choice questions, where applicable.

\section{Results}

In total, 372 participants completed the survey, of whom $62 \%$ were consultants and $34 \%$ were in training. $54 \%$ were working in an academic setting and $95 \%$ had Internet access at their workplace. In order to get a fast overview about a medical problem, physicians would prefer Google (32\%) over Wikipedia (19\%) UpToDate (18\%), or PubMed (17\%).

Medical University of Vienna, Department of Anesthesia, Critical Care and Pain M, Vienna, Austria

Full list of author information is available at the end of the article
$39 \%$ would, at least sometimes, base their medical decisions on non peer-reviewed resources. Wikipedia is used often or sometimes by $77 \%$ of the interns, $74 \%$ of residents, and $65 \%$ of consultants to get a fast overview of a medical problem. Consulting Wikipedia or Google first in order to get more information about the pathophysiology, drug dosage or diagnostic options in a rare medical condition was the choice of $66 \%, 10 \%$ or $34 \%$, respectively.

\section{Conclusions}

Certified specialists and physicians in training utilise non peer-reviewed resources and those sites impact medical decision making in acute and critical care in Austria and Australia.

\section{Authors' details}

${ }^{1}$ Medical University of Vienna, Department of Anesthesia, Critical Care and Pain M, Vienna, Austria. ${ }^{2}$ Sir Charles Gairdner Hospital, Department of Anaesthesia, Perth, Australia.

Published: 1 October 2015

\section{References}

1. LaurenT MR, Vickers TJ: Seeking health information online: does Wikipedia matter? J Am Med Inform Assoc 2009, 16(4):471-9.

2. Johnson PT, Chen JK, Eng J, Makary MA, Fishman EK: A comparison of world wide web resources for identifying medical information. Acad Radiol 2008, 15(9):1165-72.

3. Parek N, Mayer J, Rojowsky N: Connecting with Physicians Online: Searching for Answers. 2009, [12.08.2011] Available from: http://www. fdasm.com/docs/Connecting\%20with\%20Physicians\%200nline\%20Webinar\% 20Deck-\%20FINAL.PDF..

4. Hughes B, Joshi I, Lemonde H, Wareham J: Junior physician's use of Web 2.0 for information seeking and medical education: a qualitative study. Int J Med Inform 2009, 78(10):645-55.

\section{doi:10.1186/2197-425X-3-S1-A867}

Cite this article as: Rössler et al:: Influence of wikipedia and other web resources on acute and critical care decisions. a web-based surveY. Intensive Care Medicine Experimental 2015 3(Suppl 1):A867.

\section{SpringerOpen $^{\odot}$}

(C) 2015 Rössler et al.; This is an Open Access article distributed under the terms of the Creative Commons Attribution License (http:// creativecommons.org/licenses/by/4.0), which permits unrestricted use, distribution, and reproduction in any medium, provided the original work is properly cited. 